\title{
ANALYSIS OF THE EFFECT OF CARE DOSE 4D SOFTWARE USE ON IMAGE QUALITY AND RADIATION DOSE ON THE CT SCAN ABDOMEN
}

\author{
Ni Larasati Kartika Sari ${ }^{1 *}$, Merry Suzana ${ }^{2}$, Muzilman Muslim ${ }^{1}$, Dewi Muliyati ${ }^{3}$ \\ ${ }^{1}$ Universitas Nasional, Jalan Sawo Manila, Pejaten, Pasar Minggu, Jakarta Selatan 11520, Indonesia \\ ${ }^{2}$ Rumah Sakit Hermina Depok, Jl. Siliwangi No.50, Pancoran MAS, Kota Depok, Jawa Barat 16431, Indonesia \\ ${ }^{3}$ Physics Education Department, Universitas Negeri Jakarta, Jalan Rawamangun Muka, Jakarta Timur 13220, \\ Indonesia \\ *Corresponding Author Email: nilarasati@civitas.unas.ac.id
}

Received: 28 March 2020

Revised: 1 April 2020

Accepted: 4 April 2020

Online: 26 April 2020

Published: 30 April 2020

SPEKTRA: Jurnal Fisika dan Aplikasinya

p-ISSN: 2541-3384

e-ISSN: 2541-3392

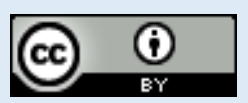

\begin{abstract}
The CT Scan is the most significant contributor to radiation dose on radiological examination, although the frequency of the examination is far below other modalities. In order to control this radiation dose, manufactures of CT Scan have equipped their units with built-in software called Automatic Exposure Control (AEC). This study aims to analyze the effect of AEC software, CARE Dose 4D, on image quality, and CTDIvol. Objects used in this study were three water phantoms, each with a diameter of $165 \mathrm{~mm}, 230 \mathrm{~mm}$, and $305 \mathrm{~mm}$. The image quality-analyzed was CT Number and noise. Measurement of image quality was carried out following Bapeten's provisions. Noise Power Spectrum (NPS) graphics were also used to further observes noise texture. The CT Number accuracy, CT Number, and noise uniformity obtained with and without CARE Dose 4D, on the three phantoms were still within Bapeten's threshold. This indicates that the use of CARE Dose 4D can still image a homogeneous object accurately. The results of the NPS curve showed that the two modes, in three phantoms, were having the same noise texture. The NPS curves also showed that the use of CARE Dose 4D produces higher noise than the non-CARE Dose 4D mode. Meanwhile, there were significant differences from the CTDIvol obtained from the two modes. The use of CARE Dose 4D software reduced dose of up to $54.34 \%$. From this, the use of CARE Dose 4D software can reduce the radiation dose while maintaining image quality.
\end{abstract}

Keywords: CT Number, CTDIvol, noise, noise power spectrum 


\section{INTRODUCTION}

The use of a CT scan in the radio-diagnostic examination is increasing. There were 13 million CT scans in the United States in 1990 and tripled to around 46 million 10 years later [1], and increased to 62 million in 2006 [2]. CT Scan accounts for the largest dose of radiation reception compared to other radiological examinations. Radiation generated on CT scans contributes $60 \%$ of radiological examinations, although the frequency of examinations is only $7 \%$ of complete examination [3]. The radiation dose received by the patient on a CT scan is 1-14 $\mathrm{mSv}$ depending on the type of examination to be carried out, proportional to the annual dose received from natural radiation sources, such as radon and cosmic radiation (1-10 mSv) depending on where a person lives [4].

Manufactures of CT Scan unit have done optimization by presenting an Automatic Exposure Control (AEC) system in the form of built-in software to reduce the radiation dose. Each manufacture's AEC systems were designed for different purposes but mainly works by modulating the tube current to compensate for variations in attenuation of the patient's body, with a different method of operator control [5,6]. Reductions in radiation dose inevitably result in a corresponding reduction in image quality. Tube current value affects the image quality, especially noise. The higher the tube current, the more number of photons is produced, thereby reducing quantum noise [7]. Therefore, users must possess a good understanding of their CT Scans' AEC systems in order to achieve dose reduction and optimal diagnostic image. An early version of CARE Dose software for pelvic CT showed that the system lowers $32 \%$ of the calculated CTDIvol with no significant loss in signal to noise ratio [8]. Evaluation of CARE Dose 4D along with other CT Scan's AEC systems showed that, for the anthropomorphic chest phantom, dose reduction with AEC mode were ranging from approximately $35 \%$ to $60 \%$, and z-axis image noise also becomes more uniform compared to the non-AEC mode [9].

Computed tomography of the abdomen and pelvis (CTAP) has become a first-line imaging method for many conditions, such as including abdominal trauma, acute abdominal pain, suspected abdominal abscess and inflammation, and in the detection and surveillance of neoplastic conditions. The image quality can, however, be limited by the patient's body build, image noise, suboptimal contrast enhancement, motion, and aliasing/blooming artifacts [10]. Therefore, it is important to evaluate whether the use of AEC in an abdominal CT Scan affects the image quality. In this study, we focused on evaluating CARE Dose 4D in reducing radiation dose and its effect on image quality of CT Number accuracy, CT Number, and noise uniformity, alongside noise texture for abdominal CT Scan.

\section{METHOD}

This study aims to analyze the effect of the use of the AEC system on a CT scan unit on image quality and CTDIvol doses. The AEC system is the default CT Scan unit software that automatically adjusts the tube current $(\mathrm{mA})$ according to variations in attenuation in the patient's body [5]. AEC adjusts attenuation at different parts when the tube surrounds the 
patient on the $\mathrm{x}$ and $\mathrm{y}$ axes and adjusts attenuation along the anatomical part of the patient's body. Thus different patient sizes will result in adjustments to the different radiation doses [11] - [13]. The current modulation of the tube can be seen in FIGURE 1.

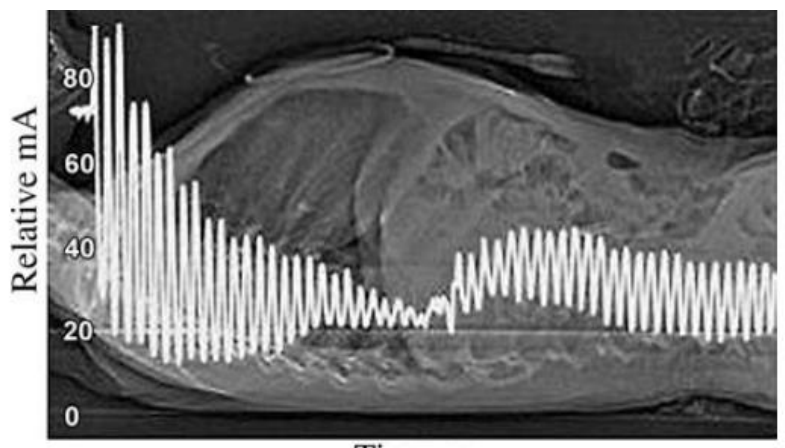

Time, $\mathrm{z}$

FIGURE 1. Graph of how AEC works in modulating tube current as a function of time (table position along the z-axis) [6]

The AEC system evaluated in this study was CARE Dose 4D software from the 16-slice Siemens Somaton emotions CT Scan. The imaging object used was three solid water phantoms, each with a diameter of $165 \mathrm{~mm}, 230 \mathrm{~mm}$, and $305 \mathrm{~mm}$. The scanning parameter settings used can be seen in TABLE 1 .

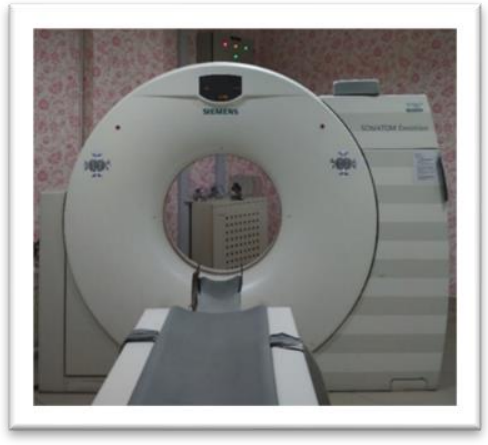

(a)

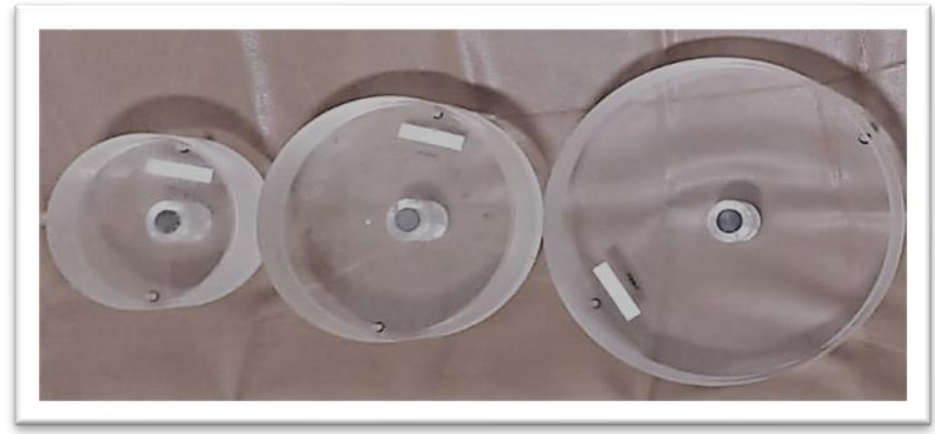

(b)

FIGURER 2. Research Tools. (a) CT Scan Siemens Somaton Emotions 16, (b) solid water phantom pf various diameters.

The image quality-analyzed is the accuracy and uniformity of CT Number and noise uniformity. CT Number is the value of the network attenuation coefficient relative to the water attenuation coefficient. CT Number varies, where the value of the CT water number is zero. The noise itself is a variation from the CT Number value. Noise is usually assessed by calculating the standard deviation of the image. Evaluation of image quality is carried out in accordance with Bapeten's recommendations in Perka Bapeten no. 2 of 2018 concerning the Conformity Test of Diagnostic and Interventional X-ray Radiology. CT Number accuracy measurement is done by making ROI (Region of Interest) in the center of the image. The 
uniformity of CT Number is measured by finding the difference between the CT Number value on the ROI at the edge and the center of the image. Meanwhile, the uniformity of noise is measured by finding the difference in the standard deviation of the ROI on edge with the center of the image. ROI placement for noise measurement can be seen in FIGURE 2. ROI number $1-4$ is the edge of the image. ROI number 5 is the center of the image.

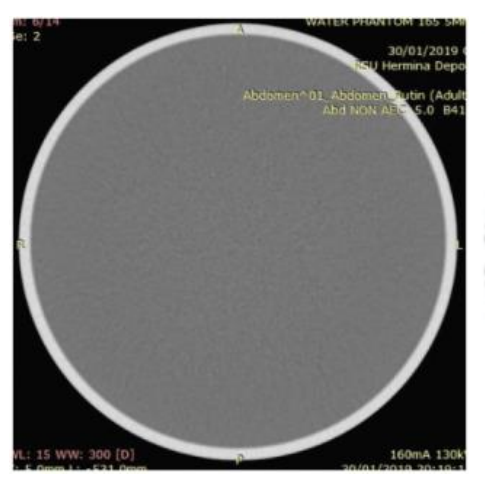

(a)

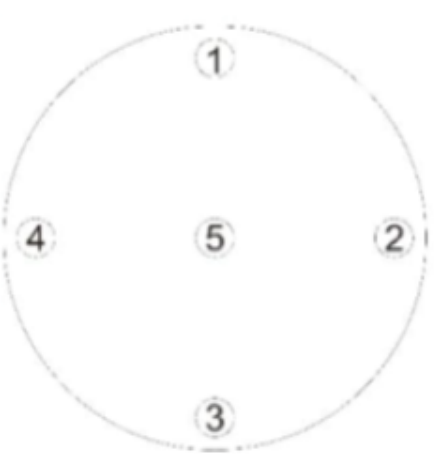

(b)

FIGURE 3. Measurement of CT Number and Noise. (a) Image examples, (b) ROI measurement points

Noise Power Spectrum (NPS) graphics are also used to further observe noise textures. NPS is a more complete description of noise than simple standard deviation. Not only able to describe the amount of noise, but NPS also describes the variation of noise as a function of spatial frequency so that it can describe the texture of noise. When combined with variable doses, it can be used for comparisons between scanners and protocols and has proven useful in translating protocols from one CT Scan platform [11]. NPS (f), for 2D images I (x, y) 2, can be formulated in EQUATION (1).

$$
N P S\left(f_{x}, f_{y}\right)=\left|\int_{x} \int_{y}[I(x, y)-I] e^{-2 \pi i\left(x f_{x}+y f_{y}\right)} d x d y\right|
$$

where $f_{x}$ is the frequency corresponding to the dimension $x$ and $f_{y}$ is the frequency corresponding to the dimension $y$, and $I$ is the average CT Number of the image.

The number of doses observed in this study was CTDIvol. CTDI or Computed Tomography Dose Index is a method of calculating the patient's average dose at one scan. CTDIvol is a measure of the average dose along the scan volume of a specific standard phantom. For adult abdomen examinations, a standard phantom has a diameter of $32 \mathrm{~cm}$. The estimated CTDIvol value used is the value indicated on the CT Scan console monitor. 
TABLE 1. Setting Scanning parameters with and without CARE Dose 4D.

\begin{tabular}{lll}
\hline Scanning Parameter & Non CARE Dose 4D & CARE Dose 4D \\
\hline $\mathrm{kV}$ & 130 & 130 \\
$\mathrm{~mA}$ & & \\
$\quad$ Water Phantom $165 \mathrm{~mm}$ & 120 & $25-120$ \\
$\quad$ Water Phantom $230 \mathrm{~mm}$ & 120 & $45-120$ \\
$\quad$ Water Phantom 305 mm & 120 & $92-120$ \\
Slice Thickness (mm) & 5 & 5 \\
Scan Time (sec) & 0.6 & 0.6 \\
Field of View (mm) & 300 & 300 \\
Pitch Factor & 0.8 & 0.8 \\
Reconstruction Algorithm & $\mathrm{B} 41 \mathrm{~s}$ & $\mathrm{~B} 41 \mathrm{~s}$ \\
\hline
\end{tabular}

\section{RESULT AND DISCUSSION}

Measurements of CT Number accuracy, CT Number uniformity, and noise uniformity were performed on the 6th slice image sample, which is the best visual image. CT Number and noise measurements are performed on the CT Scan workstation by making ROI at the center of the image, and four edge points clockwise, which is $12,3,6$, and 9. The average HU value on the ROI is CT Number, while the standard deviation value is the noise value. The results of CT Number accuracy measurements can be seen in TABLE 2. Based on these data, CT Number values, both obtained with or without CARE Dose 4D Software from the three diameter variations, nothing exceeds the Bapeten threshold, that is, still within the range $-4 \leq$ $\mathrm{CT} \leq+4$. This value shows that the use of CARE Dose 4D Software still produces accurate CT Number values, especially water.

TABLE 2. CT Number accuracy values with and without CARE Dose 4D.

\begin{tabular}{lllll}
\hline $\begin{array}{l}\text { Phanto } \\
\text { m Size } \\
(\mathrm{mm})\end{array}$ & Mode & $\begin{array}{l}\text { Average Central } \\
\text { CT Number }\end{array}$ & Threshold & Conclusion \\
\hline 165 & CARE Dose 4D & -0.4 & $-4 \leq \mathrm{CT} \leq+4$ & Passed \\
& Non & -0.4 & & \\
& CARE Dose 4D & & & \\
230 & CARE Dose 4D & -0.5 & & \\
& Non & -1.0 & & \\
305 & CARE Dose 4D & & & \\
& CARE Dose 4D & 0.6 & & \\
& Non & -0.4 & & \\
& CARE Dose 4D & & & \\
\hline
\end{tabular}


The measurement results for CT Number uniformity and noise uniformity can be seen in TABLE 3. Based on these data it is seen that in general the maximum difference between the average edge and center CT in CARE Dose 4D images is greater than Non CARE Dose 4D images, but all of them do not exceed the specified threshold by Bapeten, which is $\leq \pm 2 \mathrm{CT}$. This means that the use of CARE Dose 4D Software is still able to produce images of uniform objects well, and has a large uniform noise at all positions.

TABLE 3. CT Number and Noise uniformity values with and without CARE Dose 4D.

\begin{tabular}{cccccc}
\hline Mode & $\begin{array}{c}\text { Phantom } \\
\text { Size } \\
(\mathrm{mm})\end{array}$ & $\begin{array}{c}\text { Maximum } \\
\text { Difference in } \\
\text { Center CT \& Edges }\end{array}$ & $\begin{array}{c}\text { Maximum } \\
\text { Standard } \\
\text { Deviation of } \\
\text { Edge CT \& } \\
\text { Edge }\end{array}$ & Threshold & Conclusion \\
CARE Dose & 165 & 1.2 & 0.9 & \\
4D & 230 & 1.7 & 1.0 & $\leq \pm 2 \mathrm{CT}$ & \\
& 305 & 1.8 & 1.2 & & \\
Non CARE & 165 & 0.4 & 0.6 & \\
Dose 4D & 230 & 0.7 & 1.0 & \\
& 305 & -0.8 & 1.0 & & \\
\hline
\end{tabular}

Furthermore, to evaluate deeper noise, the Noise Power Spectrum (NPS) method is also performed. Noise evaluation using the NPS method is intended to assess the amount of noise (noise level) and texture of the noise itself. Noise can be seen broadly below the NPS chart. While noise textures can be seen in peak spatial frequency values. Low frequency spatial indicates rough noise texture, while high frequency spatial indicates smooth noise texture.

The NPS graph for each phantom with two modes can be seen in FIGURE 3. It can be seen in both modes, that the larger the phantom size, the greater the area under the NPS graph. This shows that the larger the phantom size, the greater the noise. When compared the two modes, the NPS curve with CARE Dose 4D mode has an area under the graph that is larger than the non CARE Dose 4D mode. This shows the noise level of using CARE Dose 4D is greater than without CARE Dose 4D. Both are in accordance with the evaluation of noise using standard deviations that have also been carried out. 


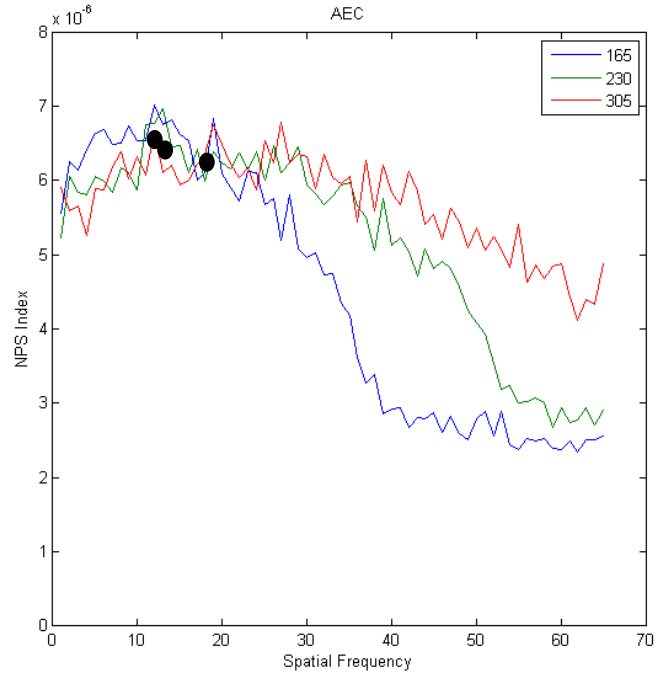

(a)

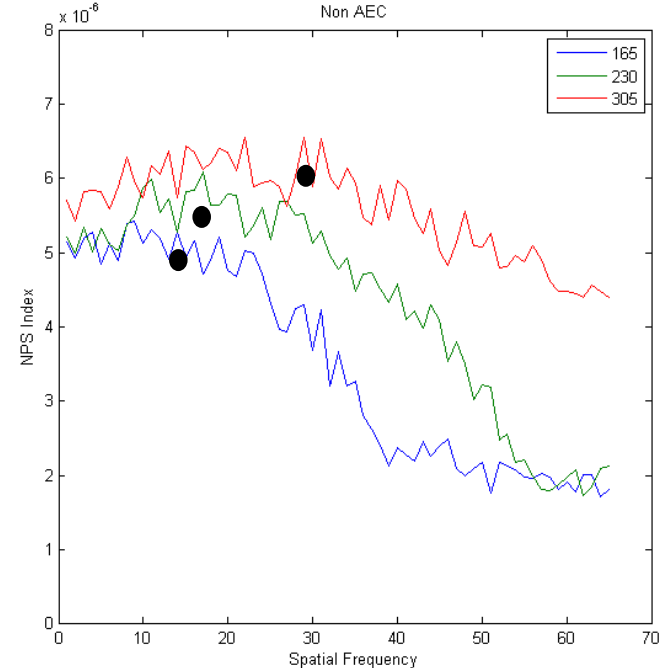

(b)

FIGURE 4. NPS Curve (a)CARE Dose 4D Mode, (b)Non CARE Dose 4D Mode.

Noise texture variations that can be seen peak spatial frequency values (marked with $\bullet$ ). In general, CARE Dose 4D and non CARE Dose 4D images produce almost the same form of noise (texture noise), i.e. the spectrum has peaks at low spatial frequencies, which means the resulting noise texture is roughly shaped. If we look at the spatial peaks of frequency at all three phantom sizes, it can be seen that the spatial frequency peaks at the size of $165 \mathrm{~mm}$ and $230 \mathrm{~mm}$ are almost the same, which means they produce almost the same noise texture. While the phantom size of $305 \mathrm{~mm}$ has a larger spatial frequency peak, which means the texture of the resulting noise is smoother.

Based on this it can be seen that CARE Dose 4D images contain greater noise than non CARE Dose 4D images. This is one of them caused by differences in the use of tube current (mA) in the two modes. Non CARE Dose 4D mode uses fixed mA, while CARE Dose 4D mode uses modulated $\mathrm{mA}$ (varies in each slice) The $\mathrm{mA}$ value affects many photons produced. Noise in radiological images is largely a quantum noise, the magnitude of which is influenced by the frequency of photons that come to the object, so the value of $\mathrm{mA}$ affects the level of noise in an image.

TABLE 4 shows the CTDIvol values with CARE Dose 4D and non CARE Dose 4D modes. Based on the table it can be seen that the CTDIvol value in non-CARE Dose 4D mode is the same in all phantom sizes. Meanwhile, CTDIvol with CARE Dose 4D mode has a different value. The larger the phantom size, the greater the CTDIvol value. When compared, the use of CARE Dose 4D can reduce the value of CTDIvol up to $54.34 \%$. This shows that the CTDIvol value only depends on the tube current value (mA) [6]. CARE Dose 4D Mode uses modulated $\mathrm{mA}$ to produce a lower dose of CTDI radiation. 
TABLE 4. CTDIvol values with and without CARE Dose 4D.

\section{Phantom Diameter (mm)}

\begin{tabular}{ccccccc} 
& \multicolumn{3}{c}{ Non CARE Dose 4D } & \multicolumn{3}{c}{ CARE Dose 4D } \\
& 165 & 230 & 305 & 165 & 230 & 305 \\
\hline CTDIvol (mGy) & 12.22 & 12.22 & 12.22 & 2.6 & 4.66 & 9.47 \\
Average & & & & & & \\
CTDIvol (mGy) & & 12.22 & & & 5.58 & \\
\hline
\end{tabular}

\section{CONCLUSION}

CT Number accuracy, CT Number, and Noise uniformity, both in CARE Dose 4D and NonCARE Dose 4D modes, are still at the Bapeten threshold. Noise evaluation with NPS shows that using CARE Dose 4D produces more noise than Non-CARE Dose 4D, but both produce almost the same noise texture. Based on the CTDIvol evaluation, it can be seen that the use of CARE Dose 4D can reduce the radiation dose up to $54.34 \%$. This shows that the use of the AEC CARE Dose 4D system is very useful in reducing the radiation dose without any affecting CT Number and noise. However, since high-quality diagnostic images are needed in an abdominal CT Scan, it's best to also evalutes the effects of the AEC system in other image quality parameters, such as spatial resolution and contrast.

\section{REFERENCES}

[1] D. J. Brenner and E. J. Hall, "Computed tomography, an increasing source of radiation exposure,” N. Engl. J. Med., 2007, vol. 357, no. 22,

[2] C. H. McCollough et al., "Strategies for Reducing Radiation Dose in CT," Radiologic Clinics of North America, 2009.

[3] Fajri et al., "Analisis Perbandingan Parameter dan Profil Dosis Menggunakan Phantom Standard dan Tidak Standar," Universitas Diponegoro, Semarang, Dikutip: https://www.youngster.physicsjurnal.com. Diakses tanggal 19 Mei 2019.

[4] B. F. Wall, "Ionising radiation exposure of the population of the United States: NCRP Report No. 160,” Radiat. Prot. Dosimetry, 2009, vol. 136.

[5] Shawna L. et al., "CARE Dose 4D CT Automatic Exposure Control System: Physics Principles and Practical Hints. Radiological Society of North America," Scientific Assembly and Annual Meeting, November 25 - November 30, 2007 ,Chicago I.L., 2007. http://archive.rsna.org/2007/5011233.html Accessed February 28, 2020.

[6] C. H. Lee et al., "Radiation Dose Modulation Techniques in the Multidetector CT Era: From Basics to Practice," Radio Graphics, 2008, vol. 28.

[7] Justin B. Solomon et al., "Relating Noise to Image Quality Indicators in CT Examinations With Tube Current Modulation," AJR, 2013, vol. 200. 
[8] G. R. Iball et al., "Assessment of tube current modulation in pelvic CT," The British Journal of Radiology, 2006, vol. 79.

[9] M. Soderberg and M. Gunnarsson, "Automatic exposure control in computed tomography - an evaluation of systems from different manufacturers," Acta Radiologica, 2010, vol. 51.

[10] K. K. Lau et al., "Imaging Techniques in Improving Image Quality of CT Abdomen: What Are the Latest?", The Royal of Australian and New Zealand College of Radiologists, 2016, https://epos.myesr.org/ranzcr/viewing/?module=viewing_poster\&pi=135764, Accessed April 5, 2020.

[11] M. Bruesewitz et al., "Smart mA - Automatic Exposure Control (AEC): Physics Principles and Practical Hints Automatic Exposure Control (AEC) Systems in CT Using AEC to Prescribe the Desired Image Quality AEC and Image Quality," Med. Educ., 2008.

[12] S. S. Mannudeep K. Kalra et al., "Techniques and Applications of Automatic Tube Current Modulation for CT1 Introduction," RSNA, 2004.

[13] C. H. McCollough and J. M. K. Michael R. Bruesewitz, "CT Dose Reduction and Dose Management Tools: Overview of Available Options," RSNA, 2006, vol. 26.

[14] J. T. Bushberg et al., "The Essential Physics of Medical Imaging," 2012, vol. 30, no. 7. 
